\title{
Theory for the nonlinear optical response of quantum-well states in ultrathin films
}

\author{
T. A. Luce and W. Hübner* \\ Institute for Theoretical Physics, Freie Universität Berlin, Arnimallee 14, D-14195 Berlin, Germany \\ A. Kirilyuk and Th. Rasing \\ Research Institute for Materials, University of Nijmegen, Toernooiveld 1, 6525 ED Nijmegen, The Netherlands \\ K. H. Bennemann \\ Institute for Theoretical Physics, Freie Universität Berlin, Arnimallee 14, D-14195 Berlin, Germany
}

(Received 25 September 1997)

\begin{abstract}
We analyze second-harmonic generation (SHG) oscillations due to quantum-well states in ultrathin overlayer films and their dependence on the electronic and magnetic structure of the film and substrate. Depending on the SHG contribution from the surface and the film-substrate interface, and the interplay between the wave-function symmetry (via the dipole matrix elements) and band-structure effects, one obtains different SHG oscillations as a function of film thickness. One may obtain only one period, which is twice that observed in the linear magneto-optical Kerr effect $\Lambda_{M}$, but also $\Lambda_{M}$ and an additional, larger period. Thus we explain, within a unified approach, recent experiments on $x$ - $\mathrm{Au} / \mathrm{Co}(0001) / \mathrm{Au}(111)$ and $x-\mathrm{Cu} / \mathrm{Fe} / \mathrm{Cu}(001)$ films, where different characteristic features of the SHG oscillations and different oscillation periods were observed. [S0163-1829(98)03212-3]
\end{abstract}

\section{INTRODUCTION}

The magnetism of metallic surfaces, thin films, and multilayer sandwiches has attracted much attention recently due to the discovery of interesting effects that also have a large potential for applications. The observation of antiferromagnetic coupling of magnetic films separated by a nonmagnetic spacer layer, ${ }^{1}$ and the subsequent discovery that this coupling could oscillate between ferromagnetic and antiferromagnetic, ${ }^{2}$ stimulated intense efforts to understand these phenomena. It was shown that in ultrathin films electronic potential discontinuities at interfaces lead to a reflection of the electronic wave functions, and consequently to a confinement of the electronic states. The resulting quantization of the perpendicular discrete components of the wave vector $\mathbf{k}_{\perp}$ gives rise to resonances in the density of states. These quantum-well states (QWS's) may act as a mediator for magnetic interlayer coupling. ${ }^{3}$

Photoemission experiments directly demonstrated oscillations of the electron density of states, ${ }^{4,5}$ and proved the existence of spin-polarized quantum-well states. ${ }^{6,7}$ However, due to the short mean free path of electrons, this method is difficult to apply for the investigation of buried interfaces and multilayers. This disadvantage is bypassed by optical techniques, since interfaces of thin metallic films are accessible by light. The linear magneto-optical kerr effect (MOKE), which is frequently used for studying magnetic properties, is sensitive to changes of the linear susceptibility $\chi^{(1)}$ as a function of the applied magnetic field, and is not interface specific. In contrast, the nonlinear optical technique of second-harmonic generation (SHG) combines a large penetration depth with a strong interface sensitivity which is derived from the breaking of inversion symmetry at interfaces and surfaces. ${ }^{8-11}$ Though the absolute nonlinear signals are small, the nonlinear magneto-optical effects are very large: compared to the linear Kerr angle, enhancements up to a factor 1000 have been observed. ${ }^{12}$

Recent experiments on $x-\mathrm{Cu} / \mathrm{Fe} / \mathrm{Cu}(001)$, $x-\mathrm{Cu} / \mathrm{Co} / \mathrm{Cu}(001)$, and $x-\mathrm{Au} / \mathrm{Co}(0001) / \mathrm{Au}(111)$ films undoubtedly proved distinct SHG oscillations, and in particular huge effects in the nonlinear magneto-optic response due to QWS's. ${ }^{13,14}$ Since the energy levels of QWS's are thickness dependent, resonant optical transitions involving QWS's caused characteristic oscillations of the SHG as a function of the film thickness. However, the observed oscillations for different film systems exhibited quite different features concerning the oscillation period, the magnitude of the magnetic SHG signal, and the dependence of the period on the incident laser frequency. ${ }^{13,14}$

In this paper we will extend our theory of the nonlinear optical response ${ }^{15,16}$ to show how SHG from ultrathin films depends on its electronic and magnetic structure and that of its substrate. As a result, we can explain within the same theoretical approach the quite different experimental SHG observations on these quantum-well systems. The theory shows the rich information contained in the various characteristic features of the SHG oscillations. In particular, it demonstrates the significance of the interplay between dipole matrix element effects, band-structure effects, and the role of the optical interference of SHG contributions from the two film interfaces. If the contributions from both the surface and interface are important, then the SHG response appears to be sensitive to the parity of the QWS's with the result that only a doubled period $2 \Lambda_{M}$ appears. Here $\Lambda_{M}$ is the period as observed in the linear Kerr effect. ${ }^{17}$ If the SHG signal is dominated by only one of the interfaces, then QWS's with even and odd symmetries contribute, and the SHG oscillations will typically reveal two periods, namely, the period $\Lambda_{M}$ and a larger period. 


\section{THEORY AND RESULTS}

In the dipole approximation the nonlinear optical response yielding the SHG intensity $I(2 \omega) \propto|P(2 \omega)|^{2}$ is given by the polarization $P_{i}(2 \omega)=\chi_{i j m}(2 \omega) E_{j} E_{m}$. Since $\chi_{i j m}$ is a polar tensor of rank 3 , it vanishes in centrosymmetric media. Only from surfaces and interfaces, where the inversion symmetry is broken, SHG arises, thus resulting in the well-known interface sensitivity of SHG. ${ }^{8-11}$ The electronic theory for the susceptibility $\chi_{i j m}$ was discussed previously. ${ }^{18}$ Here we neglect effects due to the thickness dependence of the Fresnel factors, since the optical wavelength is much larger than the film thickness. ${ }^{19}$

The surface or interface susceptibility is given by the formula ${ }^{18,20}$

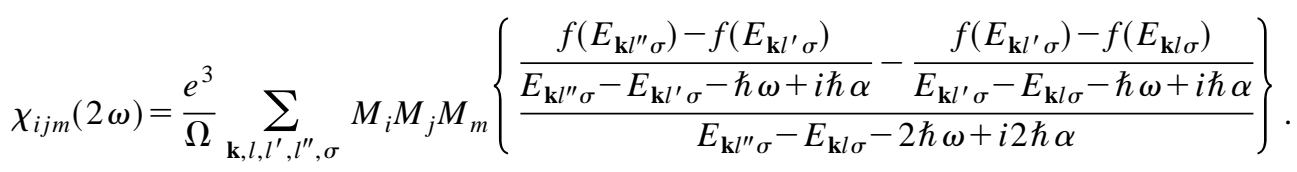

Here the symmetry of the wave functions enters through the dipole matrix elements $M_{i}=\left\langle\Psi_{k, l, \sigma}\left|p_{i}\right| \Psi_{k, l^{\prime}, \sigma}\right\rangle$, where $p_{i}$ is the momentum operator. The dependence on the electronic structure results from the eigenvalues $E_{k, l, \sigma}$, which depend on the wave vector $\mathbf{k}$, the band index $l$, and the spin $\sigma$. Note that the matrix elements may involve $s$ states, $d$ states, and QWS's and will depend on the corresponding wave functions. $f\left(E_{k, l, \sigma}\right)$ is the Fermi function, and $\alpha$ is the Lorentzian broadening of the states. Taking into account only vertical transitions, Eq. (1) already shows how changes of the susceptibility result from modifications in the joint density of states, which is probed by nonlinear optics. ${ }^{21}$ Since SHG is generated at the surface and interface of the film, the summation over the energy eigenvalues has to be performed according to the surface and interface electronic structure. For a paramagnetic material, the band structure for both spin directions is the same, and no spin dependence results. In the case of a ferromagnetic material, the nonlinear tensor $\chi_{i j m}(\mathbf{M})$ can be separated into odd $\left(\chi^{-}\right)$and even $\left(\chi^{+}\right)$ components with $\chi^{+(-)}(\mathbf{M})= \pm \chi^{+(-)}(-\mathbf{M}){ }^{22}$

Assuming for simplicity that a single tensor element $\chi_{i j l}$ dominates the SHG, one may use $\chi^{=} \chi^{+}+\chi^{-}$and $\mathbf{P}(2 \omega)=\left(\chi^{+}+\chi^{-}\right) \mathbf{E}^{2}(\omega)$, where the average effect of the Fresnel factors has been incorporated in an effective $\chi^{+}$and $\chi^{-}, \quad$ respectively. Approximately, one has $\chi_{i j l}^{-}=\chi_{i j l m} \mathbf{M}_{m}+\cdots$ and $\chi_{i j l}^{+}=\chi_{i j l}+\chi_{i j l m n} \mathbf{M}_{m} \mathbf{M}_{n}+\cdots$. In comparison with the linear MOKE, where the magnetization induces only very small off-diagonal tensor elements and is suppressed by nonmagnetic excitations, ${ }^{23} \chi^{-}$in SHG is of the same order of magnitude as the nonmagnetic tensor element $\chi^{+}$, thus inducing strong magnetic effects in SHG. ${ }^{24}$

Due to the periodic appearance of QWS's at certain energies with increasing film thickness $d$, transitions involving these QWS's are resonantly enhanced, and thus result in oscillations in SHG (and the MOKE). If the states mainly used for the optical transitions are spin polarized, the oscillations in the SHG response $I(2 \omega)$ cause corresponding oscillations in the magnetic contrast

$$
\Delta I_{-}=\frac{I(2 \omega, \mathbf{M})-I(2 \omega,-\mathbf{M})}{I(2 \omega, \mathbf{M})+I(2 \omega,-\mathbf{M})} .
$$

Here, of particular interest are contributions to $\Delta I_{-}$due to spin-polarized QWS's. Obviously, the oscillation in $\Delta I_{-}$ should be particularly strong if the electronic confinement, which leads to QWS's, acts very differently for electronic spins $\sigma$ and $-\sigma$. Due to the spin dependence of the resonant transitions, the oscillation of $I(\mathbf{M})$ and $I(-\mathbf{M})$ might be shifted somewhat with respect to each other. Then, of course, the peaks in $I(\mathbf{M})$ and $I(-\mathbf{M})$ should occur at different film thicknesses. Also, generally the behavior of $\Delta I_{-}$might be rather different if spin polarization is induced only by the substrate, or if the film itself is magnetic. For example, $\Delta I_{-}$ could rather differently weight the surface and interface as compared to $I(2 \omega)$.

If we split the susceptibility $\chi(2 \omega)$ into surface and interface contributions, $\chi^{s}(2 \omega)$ and $\chi^{i}(2 \omega)$, respectively, then

$$
\chi_{i j m}(2 \omega)=\chi_{i j m}^{s}(2 \omega)+\chi_{i j m}^{i}(2 \omega) .
$$

Hence, depending on the ratio of the interface vs the surface contribution, one obtains that the SHG oscillations resulting from $\chi_{i j m}$ are dominated by the symmetry of the dipole matrix elements and thus the optical interference between the two contributions $\chi^{s}$ and $\chi^{i}$. If the SH intensity has equal contributions from the surface and interface, then according to Eq. (3) one obtains no SHG signal for $\chi^{s} \chi^{i}=-\left(\chi^{s}\right)^{2}$. If the SH intensity is essentially determined by either one of the two contributions to $\chi_{i j m}$, then the relative phases of $\chi_{i j m}^{s}(\omega)$ and $\chi_{i j m}^{i}(\omega)$ and their interference is not important; ${ }^{25}$ see Fig. 1(a). In that case the energy eigenvalues and dominant optical transitions reflecting the electronic structure are most important for the SHG.

The SHG intensity and the period of the SHG oscillations are determined by the region of $\mathbf{k}$ space contributing to the SHG signal, as is obvious from Eq. (1). For electronic structures whose optical spectra are dominated by a small region in $\mathbf{k}$ space, SHG is highly $\mathbf{k}$ selective. The structure of the SHG oscillation as a function of the film thickness $d$ is spiky and pronounced, since a few resonant transitions strongly determine the SHG due to the high density of states at these $\mathbf{k}$ points. Figure 2 illustrates this situation for the case of the (100) surface of $\mathrm{Cu}$, where contributions of the $\mathbf{k}$ points in the neighborhood of the $\Gamma$ point mostly dominate the SHG. A possible nonlinear transition is indicated by the arrow. On the other hand, if a larger $\mathbf{k}$-space volume is contributing equally to the SHG signal, the SHG spectrum of the film system has a less sharp structure. An example of this case is 

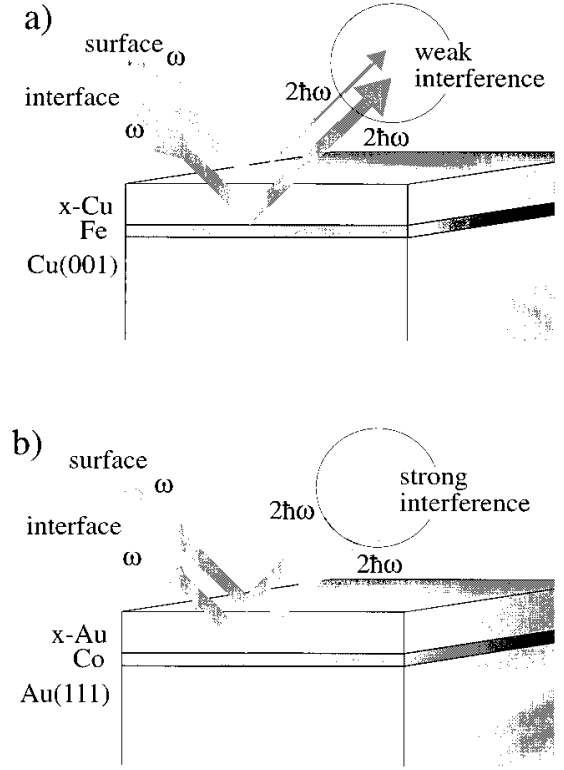

FIG. 1. Illustration of the two contributions from the surface and interface to SHG from thin films with QWS's. (a) Weak interference, since either the interface (as indicated) or the surface contribution dominates. (b) Both contributions are nearly equal, resulting in strong interference effects.

given in Fig. 3, where the electronic structure for the (111) surface of $\mathrm{Cu}$ is sketched. Here a larger region of $\mathbf{k}$ points contributes uniformly to the optical response.

To simplify the discussion, we separately treat film systems where optical interference effects due to $\chi^{s}$ and $\chi^{i}$ are negligible, and where $\chi^{s}$ and $\chi^{i}$ both contribute. In the first case we again analyze separately the situation when the QWS's are dominantly involved as final states, as intermediate states, and finally when both final and intermediate states together cause SHG oscillations. ${ }^{26}$

\section{A. One dominating interface contribution}

First, we analyze the situation where no destructive interference of the surface and interface contributions to SHG

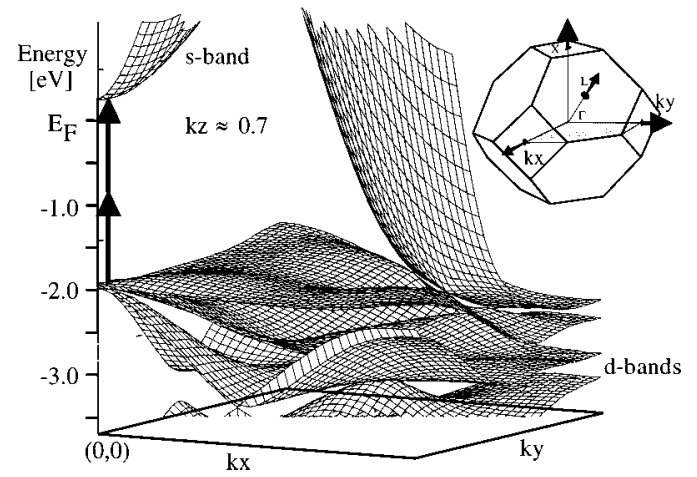

FIG. 2. The energy bands for $\mathrm{Cu}$ for a plane parallel to the indicated one in the fcc Brillouin zone. The normal of the plane is in the $\Gamma-X$ direction, corresponding to a (100) surface. The energy bands for the (100) surface at $\mathbf{k}_{\perp} \approx 0.7$ (in units of the Brillouinzone length) are drawn. The bands at $\left(k_{x}, k_{y}\right)=(0,0)$ dominate the optical spectrum due to their small gradient there. The dominant SHG transitions are indicated by the arrow.

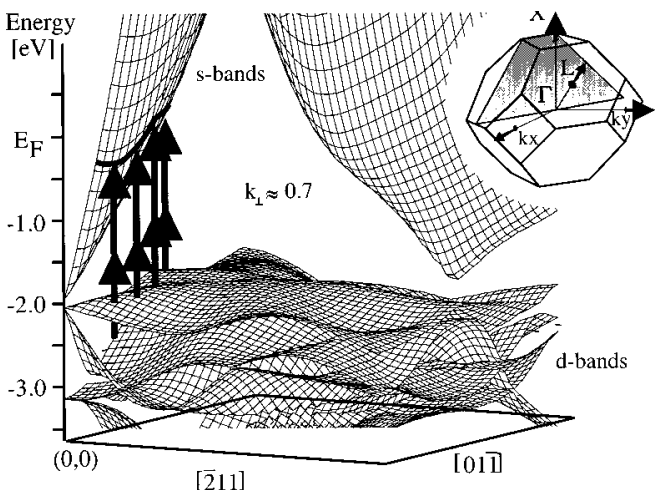

FIG. 3. The calculated energy bands for $\mathrm{Cu}$ for a plane parallel to the indicated one in the fcc Brillouin zone. The plane corresponds to a (111) surface of $\mathrm{Cu}$. Its normal is the $\Gamma-L$ direction. The energy bands for the (111) surface at $\mathbf{k}_{\perp} \approx 0.7$ (in units of the Brillouin-zone length) are sketched. Apparently, no region with comparable weight as in Fig. 2 is present, and consequently $\mathbf{k}$ selectivity is less pronounced. Note that various $\mathbf{k}$ points contribute equally to the optical response. Some possible optical transitions are indicated by arrows.

occurs, or where $\chi^{s}$ or $\chi^{i}$ dominantly contribute to SHG. If, above the Fermi energy, QWS's are mainly available for optical transitions, then a first peak in SHG appears for a film thickness $d_{1}$ at which a QWS has moved across $E_{F}$ and becomes available as final state. Then, again, a SHG peak results for a film thickness $2 d_{1}$ at which the previous situation is repeated, and so on; see Fig. 4 for illustration. The resulting oscillation period $\Lambda_{1}$ is the same as that calculated for the MOKE $\left(\Lambda_{M}\right)$ at a fundamental frequency $\hbar \omega=1.6 \mathrm{eV}$, and must be longer than or equal to the period observed in photoemission, when only QWS effects at the energy $E_{F}$ are investigated. ${ }^{5}$ Note that, in the MOKE and SHG periods the QWS's above the Fermi level may play a role if corresponding optical transitions occur. ${ }^{27} \Lambda_{1}=\Lambda_{M}$ can be deduced from the electronic structure as the ratio $\Lambda_{M}=\left[\mathbf{k}_{\mathrm{BZ}} /\left(\mathbf{k}_{\mathrm{BZ}}-\mathbf{k}_{1}\right)\right]$, where $\mathbf{k}_{1}$ is the wave vector at which the most important resonances in the MOKE occur. If now, for increasing film thickness $d>d_{1}$, not only the first QWS above $E_{F}$, but also the next higher one, becomes available for an optical transition (see Fig. 4), then a second peak in the SHG response occurs and consequently a second period appears. This second period $\Lambda_{2}$ also depends, of course, on the band structure, and thus gives additional information about the available final and intermediate states. Note that $\Lambda_{2}$ is independent of $\Lambda_{M}$, and may only by accident be equal to $2 \Lambda_{M}$. Of course, the occurrence of additional periods depends on the band structure. For a photon energy of $1.6 \mathrm{eV}$ there are no further intermediate states leading to resonant nonlinear transitions, and thus no additional oscillation periods occur. If the photon frequency is such that many periods may occur, then it may happen that the resultant SHG signal exhibits only a washed-out oscillatory behavior.

As is obvious from Fig. 4, if only a few dominant optical transitions occur, the $\mathbf{k}$ selectivity of the optical response is optimal, and the SHG oscillations will be most pronounced. Of course, the strength of the absolute signal depends further on the joint density of states for optical transitions. Furthermore, the SHG oscillation will strongly change with the in- 




FIG. 4. Illustration of the generation of SHG oscillations due to QWS's if optical interference of SHG from surface and interface is negligible. The electronic structure refers to the case of a $x \mathrm{Cu} / \mathrm{Fe} /$ $\mathrm{Cu}(100)$ system. Since the $\Gamma-X$ direction dominates the SHG generation (see Fig. 2), only this section of the Brillouin zone is considered. Only the rightmost QWS's of a $12 \mathrm{ML} \mathrm{Cu}$-film are drawn (dots). Note that for $x=6$ ML QWS's (b) and (d) are not present. Possible dominant transitions involving QWS's as final states are indicated as (i) and (ii). Whereas transition (i) is possible for films with 6 and 12 ML leading to an oscillation period of $6 \mathrm{ML}$, transition (ii) is possible only for $12 \mathrm{ML}$. $\mathbf{k}_{1}$ and $\mathbf{k}_{2}$ denote the $\mathbf{k}_{\perp}$ values along $\Gamma-X$ which determine the oscillation periods $\Lambda_{1}$ and $\Lambda_{2}$, respectively. A possible optical transition involving an occupied QWS as an intermediate state is indicated. The resultant SHG oscillations due to occupied QWS's generally have a different period in comparison with (i) and (ii).

cident laser frequency $\omega$, since $\hbar \omega$ must fit the optical transitions. According to Eq. (1) this frequency dependence is strongest if the matrix elements are less dominant than the energy denominators in the susceptibility $\chi$.

Note that for different incident laser frequencies different $\mathbf{k}$ points dominate the nonlinear optical response. Then it is of course necessary that confinement be present at these $\mathbf{k}$ points, to obtain oscillations in the SHG signal.

In the case of a ferromagnetic spacer layer between the substrate and the overlayer film, resonant transitions for minority and majority electrons are possible at different overlayer thicknesses due to the spin-split $d$ states. Thus a phase shift for the $I(\mathbf{M})$ and $I(-\mathbf{M})$ signals occurs. Consequently one may observe an enhanced magnetic contrast of the SHG response.

Figure 4 describes the situation when matrix elements and optical interference effects do not dominate. Obviously, one expects a sensitive dependence of the QWS oscillations in SHG on the frequency of the incoming light. For transitions (i) and (ii) sketched in Fig. 4, resulting in SHG oscillations mainly due to QWS's above $E_{F}$, one expects that the periods $\Lambda_{1}$ and $\Lambda_{2}$ increase as $\hbar \omega$ increases, since for increasing frequency the resonant transition indicated by (ii) occurs for a QWS appearing at a higher energy and thus at a larger $\mathbf{k}_{\perp}$. This corresponds to an increasing period for increasing $\hbar \omega$. Obviously, $2 \Lambda_{1} \simeq \Lambda_{2}$ if $\left(\mathbf{k}_{\mathrm{BZ}}+\mathbf{k}_{1}\right) / 2 \simeq \mathbf{k}_{2}$.

If QWS's are formed in an electronic band below $E_{F}$ [transition (iii) in Fig. 4], SHG oscillations result again due to changes in the density of states caused by the QWS's upon varying film thickness. In contrast to the previous case with QWS's above $E_{F}$, the oscillation period may increase for decreasing photon frequency.

If now QWS's below and above $E_{F}$ cause oscillations, a period consisting of a superposition of the two periods results, as proposed already by Straub and co-workers. ${ }^{26,28}$ Depending on the weight of these two oscillations, it is possible that the resulting single period increases as the photon frequency decreases, for example. This may explain the behavior observed for $x-\mathrm{Cu} / \mathrm{Co} / \mathrm{Cu}(001)$ by Vollmer and co-workers. ${ }^{29,30}$

\section{B. Two interfering interface contributions}

Second, we analyze the case where optical interference effects play a dominant role. Due to $I(2 \omega) \propto\left|\chi(2 \omega)_{i j m}\right|^{2}$ the relative phase of the two terms $\chi_{i j m}^{s}(2 \omega)$ and $\chi_{i j m}^{i}(2 \omega)$ is important, and may cause a cancellation of these two contributions. This is the situation illustrated in Fig. 1(b). The symmetry of the dipole matrix elements is now essential if optical interference of the two terms in Eq. (3) occurs. Obviously, the interference is strongest if the two SHG contributions at the surface and interface have nearly the same weight. Using for simplicity $\left|\chi_{i j m}^{s}(2 \omega)\right| \approx\left|\chi_{i j m}^{i}(2 \omega)\right|$, one obtains

$$
I(2 \omega) \approx 2\left|\chi_{i j m}^{s}(2 \omega)\right|^{2}+2 \chi_{i j m}^{s}(2 \omega) \chi_{i j m}^{i}(2 \omega) .
$$

For equally weighted contributions one obviously obtains a perfect cancellation of the thickness-dependent $\chi(2 \omega)$, if the resultant phase of the product of $\chi_{i j m}^{s}(2 \omega) \chi_{i j m}^{i}(2 \omega)$ is -1 , like for a phase shift $\pi$ at the interface or for inversion symmetric films [due to the transformation property of $\chi_{i j m}^{s}(2 \omega)$ under inversion, $\left.\chi_{i j m}^{s}(2 \omega) \rightarrow-\chi_{i j m}^{i}(2 \omega)\right]$.

Obviously, the parity of the QWS's also plays an important role for $I(2 \omega)$, if the surface contribution to SHG is nearly equal to the interface contribution. For example, if for increasing film thickness $d$ (at $d=d_{1}$ ) the first unoccupied QWS close to the Fermi energy $E_{F}$, which sets the oscillation period, has even parity (see Fig. 5), and if at the interface no phase shift of $\pi$ occurs, then no SHG results, ${ }^{14}$ since the product of the three dipole transition matrix elements is small in contrast to the case of a QWS with odd parity. Thus, in the case where the symmetry of the QWS regulates $I(2 \omega, d)$, one should observe in SHG a pronounced period doubling of the oscillation period, since only the QWS with odd parity causes oscillations. This is also true for a possible larger second period, that, however, will be difficult to detect experimentally.

In detail this period doubling of the SHG oscillations due to QWS's can be understood if one investigates the matrix element product in Eq. (1). Assuming for example that only one QWS is involved, then one has for a typical SHG transition for the product of the matrix elements

$$
\langle\mathrm{d}|\mathbf{p}| \mathrm{d}\rangle\langle\mathrm{d}|\mathbf{p}| \mathrm{QWS}\rangle\langle\mathrm{QWS}|\mathbf{p}| \mathrm{d}\rangle \text {. }
$$

Expanding the $d$ states in terms of Wannier functions, using for simplicity approximately the even symmetry of the corresponding atomic wave functions, and further taking into account the odd symmetry of the momentum operator $\mathbf{p}$, for 
a)

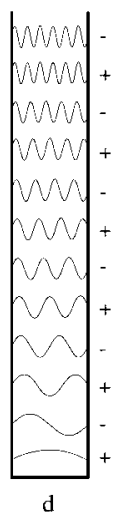

b)

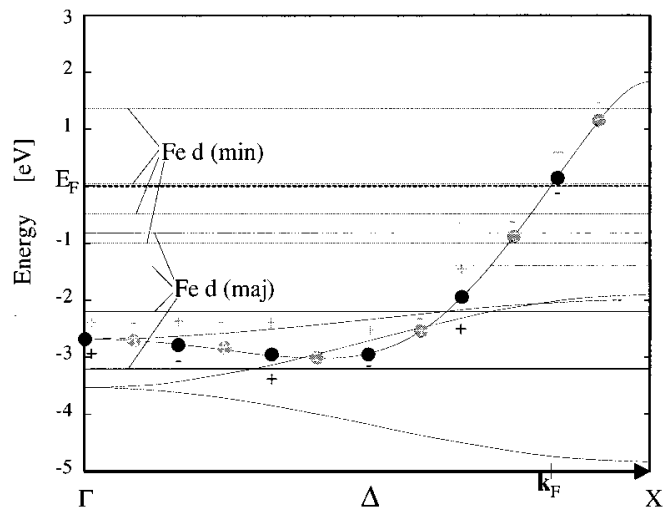

FIG. 5. (a) Wave functions for a particle in a box of thickness $d$ with infinite walls as a model for QWS wave functions. The symmetry of the QWS alternates with increasing quantum number. (b) Using this model, the symmetry of the QWS is indicated for 6 (only full black dots; parity sign of the QWS as indicated) and $12 \mathrm{ML}$ (full and grey dots; parity sign of the QWS as indicated) is sketched. Obviously the parity of the QWS at $E_{F}$ changes sign. Thus, if the plus sign causes constructive interference between surface and interface layers at $12 \mathrm{ML}$, the minus sign for $6 \mathrm{ML}$ causes destructive interference. For surface and interface contributions of approximately equal weight, no SHG will be detectable. Thus a doubling of the MOKE period $\Lambda_{M}$ occurs.

an odd QWS one obtains that only the first matrix element is small. In contrast, for an even QWS all three matrix elements are small. ${ }^{31}$ Thus, if surface and interface contribute, the signal from an even QWS is negligible compared to the one from an odd QWS. If using Bloch functions for the $d$ states, one has to keep in mind that transitions involving QWS's take place at specific $\mathbf{k}$ values. For these $\mathbf{k}$ values the Bloch

functions in the film also have definite symmetry properties, and the same argument as above is valid. ${ }^{32}$

The minimum between two peaks of $I(2 \omega, d)$ is much deeper due to the vanishing matrix element product (the phase difference due to the thickness increase is negligible compared to the wavelength of light). If the spin polarization of the QWS is not very pronounced, one expects only a relatively small magnetic contrast $\Delta I_{-}$in SHG, since the $d$ states of a ferromagnetic spacer are not strongly involved in the transitions contributing to SHG. (Otherwise there would be no equally weighted contribution from surface and interface.)

Generally, one notes that if the system under consideration shows only a weak k selectivity, the SHG response will display smooth oscillations without strong peaks and almost no frequency dependence, since the wavelength dependence of the period is washed out by the slightly different periods generated from the different $\mathbf{k}$ points contributing to the optical transitions. Of course, when both the symmetry of the dipole matrix elements and the electronic structure of the (magnetic transition metal) substrate are taken into account, the SHG signal will be more complex. However, a strong $\mathbf{k}$ selectivity is due to the influence of the substrate, which makes additional states available for resonant transitions. Then there can be no equally weighted contributions from interface and surface, and interference effects should be small.

Note that only the QWS's show a strong dependence on the layer thickness, in contrast to the other bandlike states of the film. Thus it is sufficient for the thickness dependence of SHG to consider transitions where QWS's are involved. However, there are also other contributions to SHG, e.g., involving only $d$ states. These transitions create only a background contribution to SHG. The strong SHG oscillations result from transitions involving QWS's.

TABLE I. Characteristics of SHG response, and its dependence on the electronic structure for thin films.

\begin{tabular}{|c|c|c|}
\hline & $\left|\chi^{i}\right| \gg\left|\chi^{s}\right|$ & $\left|\chi^{i}\right| \approx\left|\chi^{s}\right|$ \\
\hline k selectivity & $\begin{array}{l}{[\mathrm{x}-\mathrm{Cu} / \mathrm{Fe} / \mathrm{Cu}(001) \text {, for example] }} \\
\text { - strong magnetic signal due to strong } \\
\text { (magnetic) interface contributions } \\
\text { - sharp SHG peaks due to few contributing } \\
\mathbf{k} \text { points, resulting in strong resonances } \\
\text { - strong frequency dependence of the SHG } \\
\text { oscillation period due to the dispersion of } \\
\text { QWS's in the } \mathbf{k}_{\perp} \text { direction } \\
\text { - MOKE period and larger periods visible; } \\
\text { no exact doubling of the MOKE period }\end{array}$ & $\begin{array}{l}\text { - weak SHG signal, from only a few } \mathbf{k} \text { points, } \\
\text { and without strong interface contributions } \\
\text { - doubled period, and additional periods } \\
\text { are frequency dependent } \\
\text { - MOKE period absent; doubled and additional } \\
\text { SHG periods visible }\end{array}$ \\
\hline no $\mathbf{k}$ selectivity & $\begin{array}{l}\text { - strong magnetic signal, due to a strong interface } \\
\text { contribution } \\
\text { - broad SHG peaks, since contributions } \\
\text { come from many k points } \\
\text { - weak frequency dependence of the oscillation } \\
\text { period } \\
\text { - MOKE period and larger periods present }\end{array}$ & $\begin{array}{l}\text { [x-Au/Co(0001)/Au(111), for example] } \\
\text { - smaller magnetic contribution, since interface } \\
\text { and (nonmagnetic) surface contributions } \\
\text { are of same magnitude } \\
\text { - broad, smooth peaks, since interference } \\
\text { effects do not change abruptly } \\
\text { - SHG oscillation period is rather independent } \\
\text { of the frequency, since the SHG signal } \\
\text { is caused by the QWS's near } E_{F} \\
\text { - MOKE period absent, doubled period } \\
\text { present }\end{array}$ \\
\hline
\end{tabular}


In Table I we list characteristic features present if matrix element effects are dominant or negligible, respectively, and for more or less dominant $\mathbf{k}$ selectivity. Of course, due to the electronic structure of the film, which may be different at its surface, and the substrate interface, the above two limiting situations may not be realized, and then the QWS features in SHG are less pronounced.

\section{EXAMPLES}

In this section we want to demonstrate the significance of the wave-function symmetry and the band structure for the SHG response for two quite different systems that were investigated recently. First we discuss $x-\mathrm{Cu} / \mathrm{Fe} / \mathrm{Cu}(001) .^{13,16}$ Here, we find that the essential contribution comes just from one interface, and that it also has strong $\mathbf{k}$ selectivity. For this system it is sufficient to restrict $\mathbf{k}$ to $\left(k_{x}, k_{y}\right)=(0,0)$, since the Fermi surface has a caliper and a large density of states (DOS) at $E_{F}$. Furthermore, from an inspection of the band structure in the $\mathbf{k}_{\perp}$ direction, one finds that three different types of optical transitions essentially contribute to $\chi_{i j m}(2 \omega)$ ( $\mathbf{p}$ is the dipole operator):

$$
\begin{aligned}
& \text { (i) }\langle\mathrm{Fe}|\mathbf{p}| \mathrm{Fe}\rangle\langle\mathrm{Fe}|\mathbf{p}| \mathrm{QWS}\rangle\langle\mathrm{QWS}|\mathbf{p}| \mathrm{Fe}\rangle, \\
& \text { (ii) }\langle\mathrm{Cu}|\mathbf{p}| \mathrm{Fe}\rangle\langle\mathrm{Fe}|\mathbf{p}| \mathrm{QWS}\rangle\langle\mathrm{QWS}|\mathbf{p}| \mathrm{Cu}\rangle, \\
& \text { (iii) }\langle\mathrm{Cu}|\mathbf{p}| \mathrm{Cu}\rangle\langle\mathrm{Cu}|\mathbf{p}| \mathrm{QWS}\rangle\langle\mathrm{QWS}|\mathbf{p}| \mathrm{Cu}\rangle \text {. }
\end{aligned}
$$

The optical transitions corresponding to (i) and (ii) are responsible for the dominant $\mathrm{Fe} / \mathrm{Cu}$ interface contribution to SHG due to QWS's, since the important $d$ states and the large local DOS of Fe are not present at the $\mathrm{Cu}$-film surface. The surface contribution to SHG only involves the matrixelement combination (iii). Thus the $\mathrm{Fe} / \mathrm{Cu}$ interface dominates the SHG, and essentially $\chi_{i j m}(2 \omega) \approx \chi_{i j m}^{i}(2 \omega)$. As a consequence, one expects a strong magnetic contrast $\Delta I_{-}$ due to the spin-split $\mathrm{Fe} d$ bands at the magnetic interface. This is in perfect agreement with experiment, ${ }^{13}$ that showed a strong magnetic contrast and two wavelength-dependent oscillation periods $\Lambda_{1}=\Lambda_{M}$ and a larger one $\Lambda_{2}$. The latter dominates the SHG signal.

The wavelength dependence can also be deduced from our model. For unoccupied QWS's dominating the thickness dependence of SHG, one would expect an increase of the period with decreasing wavelength. ${ }^{16}$ However, if occupied QWS's contribute considerably, the period resulting from a superposition of both contributing types of QWS's below and above $E_{F}$ may cause a decrease of the period with decreasing wavelength, as is observed in experiment. ${ }^{26}$

Note that our calculation in Ref. 16 yields no oscillatory effect from occupied QWS's. This may be due to the use of a simplified electronic structure. It would be of interest to see whether a more realistic band structure of the film would also yield oscillatory effects due to occupied QWS's. ${ }^{33}$

As an example for systems with dominant contributions from both the surface and interface, we consider $x-\mathrm{Au} / \mathrm{Co}(0001) / \mathrm{Au}(111) .{ }^{14}$ From the band structure one can deduce that the Co states cause confinement for majority states above approximately $(-1) \mathrm{eV}$ below $E_{F}$. Since the $2.38-\mathrm{eV}$ onset of $d$-band transitions comes from states around the neck of the Fermi surface near the $L$ point, ${ }^{34}$



b)



FIG. 6. (a) Regions in the irreducible Brillouin zone with $s$ states having considerable weight for the formation of SHG oscillations due to QWS's. The $\Gamma$ point is at the center of the Brillouin zone (hidden by the $L$ point). Along the indicated $\mathbf{k}$ directions (region A) there are states acting as final states. If QWS's are present in these regions, they give rise to a strong SHG output. QWS's occurring in other regions (e.g., in the $\Gamma$ - $L$ direction) do not contribute significantly since the QWS's are then too far above $E_{F}$. (b) Illustration of the $\mathrm{Au}$ and $\mathrm{Co}$ band structures for $\mathbf{k}_{\perp}$ corresponding to the direction perpendicular to the surface for the $x-\mathrm{Au} / \mathrm{Co}(0001) / \mathrm{Au}(111)$ system. Confinement for the majority electrons of $\mathrm{Au}$ is possible for energies $>-1 \mathrm{eV}$ due to the lacking overlap between the Au and Co bands. However, the possible Au QWS's there are too high above $E_{F}$ to be reached by photons of $\hbar \omega$ less than $1.7 \mathrm{eV}$.

these directions have to be considered. However, since there is no caliper of the Fermi surface, contributions from many $\mathbf{k}$ points have to be taken into account. The area of contributing k points is indicated in Fig. 6(a) (region A). This causes only a weak $\mathbf{k}$ selectivity, and consequently the SHG peak with respect to the Au layer thickness is relatively broad. Although not only the directions $\Gamma-L$, but also parallel ones matter, one may again estimate the average MOKE period $\Lambda_{M} \approx 7 \mathrm{ML}$ approximately from the Au band structure. ${ }^{35}$

From inspection of the Co band structure sketched in Fig. 6(b), it can be seen that there are no Co $d$ states serving as intermediate states. Thus the interface and surface contribution should be very similar, which leads to a strong influence of the QWS parity on the SHG contribution, and consequently to a doubling of the $\Lambda_{M}$ period $\Lambda_{1}=2 \Lambda_{M}$. This is in excellent agreement with experiment, where a period of 13 ML was observed. ${ }^{14}$ An even larger period $\Lambda_{2}$ is, due to the period doubling, expected to be in the range of more than 20 $\mathrm{ML}$, and is therefore hardly detectable.

The magnetic SHG signal can be understood as follows. Since mainly QWS's for majority electrons occur, the magnetic SHG signal should show the same oscillation period as the nonmagnetic SHG signal. This is in excellent agreement 
with experiment. ${ }^{14}$ The oscillation period for the total SHG response and for the magnetic contrast equals 13 ML for $p$ polarization of both the incident $\omega$ and the outgoing reflected $2 \omega$ beam. The linear MOKE period is found to be 7 ML. Thus the period doubling indicates that in the $x-\mathrm{Au} / \mathrm{Co} / \mathrm{Au}(111)$ system the Co states are not strongly involved as initial states, but necessary for the formation of the confinement of the Au states. A further indication for the minor influence of the Co $d$ states is the relatively small magnetic signal $(-5 \%$ to $+20 \%)$ compared to the one in the $x-\mathrm{Cu} / \mathrm{Fe} / \mathrm{Cu}(001)$ system $(-50 \%$ to $+50 \%)$, and the absence of any dependence of the observed period on the incident laser frequency, which shows that no $\mathbf{k}$ points with resonances involving Co states dominate the spectrum. ${ }^{36}$

\section{CONCLUSION}

In summary, we have shown, by theoretical calculations supported by experimental results, the rich information that can be obtained from SHG of ultrathin films. Our analysis shows that rather different experimental observations of oscillating SHG signals due to the presence of QWS's (Refs. 13 and 14) can be understood within the same theoretical framework. Clearly, since more optical transitions are in-

*Present address: Max-Planck-Institut für Mikrostrukturphysik, Weinberg 2, 06120 Halle, Germany.

${ }^{1}$ P. Grünberg, R. Schreiber, Y. Pang, M. B. Brodsky, and H. Sowers, Phys. Rev. Lett. 57, 2442 (1986).

${ }^{2}$ S. S. P. Parkin, N. More, and K. P. Roche, Phys. Rev. Lett. 64, 2304 (1990).

${ }^{3}$ M. D. Stiles, Phys. Rev. B 48, 7238 (1993).

${ }^{4}$ J. E. Ortega and F. J. Himpsel, Phys. Rev. Lett. 69, 844 (1992).

${ }^{5}$ J. E. Ortega, F. J. Himpsel, G. J. Mankey, and R. F. Willis, Phys. Rev. B 47, 1540 (1993).

${ }^{6}$ C. Carbone, E. Vescovo, O. Rader, W. Gudat, and W. Eberhardt, Phys. Rev. Lett. 71, 2805 (1993).

${ }^{7}$ K. Garrison, Y. Chang, and P. D. Johnson, Phys. Rev. Lett. 71, 2801 (1993).

${ }^{8}$ Y. R. Shen, The Principles of Nonlinear Optics (Wiley, New York, 1984).

${ }^{9}$ Th. Rasing, Appl. Phys. A: Solids Surf. 59, 531 (1994).

${ }^{10}$ T. F. Heinz, in Nonlinear Surface Electromagnetic Phenomena, edited by H. E. Ponath and G. I. Stegeman (North-Holland, Amsterdam, 1991), p. 335

${ }^{11}$ G. L. Richmond, J. M. Robinson, and V. L. Shannon, Phys. Solid State 28, 1 (1988).

${ }^{12}$ B. Koopmans, M. Groot Koerkamp, Th. Rasing, and H. van den Berg, Phys. Rev. Lett. 74, 3692 (1995).

${ }^{13}$ M. Straub, R. Vollmer, and J. Kirschner, Verh. Dtsch. Phys. Ges. 6, 1595 (1996), and private communication.

${ }^{14}$ A. Kirilyuk, Th. Rasing, R. Megy, and P. Beauvillain, Phys. Rev. Lett. 77, 4608 (1996).

${ }^{15}$ W. Hübner, Phys. Rev. B 42, 11553 (1990).

${ }^{16}$ T. A. Luce, W. Hübner, and K. H. Bennemann, Phys. Rev. Lett. 77, 2810 (1996).

${ }^{17}$ R. Megy, A. Bounouh, Y. Suzuki, P. Beauvillain, P. Bruno, C. Chappert, B. Lecuyer, and P. Veillet, Phys. Rev. B 51, 5586 (1995)

${ }^{18}$ U. Pustogowa, W. Hübner, and K. H. Bennemann, Phys. Rev. B 48, 8607 (1993). volved, $(M)$ SHG can reveal more information than MOKE. Generally, one may obtain an oscillatory behavior of the SHG intensity for increasing film thickness $d$, which is dominantly controlled either by the optical interference and the symmetry of the dipole matrix elements or, if interference effects are negligible, by the energy spectrum and strong optical transitions controlled by the light energy $\hbar \omega$. The period of the SHG oscillations due to QWS's may change characteristically with photon frequency, thus revealing prominent optical transitions and $\mathbf{k}$ selectivity, in which QWS's are involved. For other film structures one may easily derive corresponding conclusions using the arguments presented in this paper. For layered film structures with QWS's in several films, one expects interesting interference effects which may also reflect the magnetic structure of the multilayer structure.

\section{ACKNOWLEDGMENTS}

We thank J. Kirschner for useful information and discussions. Part of this work was supported by the TMR Network NOMOKE (ERBFMRXCT960015) and by Deutsche Forschungsgemeinschaft, SFB 290.

${ }^{19}$ W. Hübner, K. H. Bennemann, and K. Böhmer, Phys. Rev. B 50, 17597 (1994).

${ }^{20}$ U. Pustogowa, W. Hübner, K. H. Bennemann, and T. Kraft, Phys. Rev. B 102, 109 (1997).

${ }^{21}$ Since in nonlinear optics three states are involved, the joint density of states is related to all three states.

${ }^{22}$ Ru-Pin Pan, H. D. Wei, and Y. R. Shen, Phys. Rev. B 39, 1229 (1989).

${ }^{23}$ U. Pustogowa, W. Hübner, and K. H. Bennemann, Phys. Rev. B 49, 10031 (1994).

${ }^{24}$ Th. Rasing, in Nonlinear Magneto-Optics, edited by K. H. Bennemann (Oxford University Press, London, in press).

${ }^{25}$ Generally, for thick films one expects that the interface contribution to SHG becomes less important.

${ }^{26}$ M. Straub, R. Vollmer, and J. Kirschner, Verh. Dtsch. Phys. Ges. 7, 818 (1997).

${ }^{27}$ P. Bruno, Y. Suzuki, and C. Chappert, Phys. Rev. B 53, 9214 (1996).

${ }^{28}$ M. Straub, Ph.D. thesis, Max-Planck-Institute für Mikrostrukturphysik Halle, Germany, 1997.

${ }^{29}$ R. Vollmer, H. Regensburger, M. Straub, and J. Kirschner, Verh. Dtsch. Phys. Ges. 7, 800 (1997).

${ }^{30} \mathrm{R}$. Vollmer, in Nonlinear Magneto-Optics (Ref. 24).

${ }^{31}$ If both surface and interface contribute nearly equally to SHG, then, for example, one may write $\langle d|\mathbf{p}| \mathrm{QWS}\rangle \approx\langle d|\mathbf{p}| \mathrm{QWS}\rangle_{s}+\langle d|\mathbf{p}| \mathrm{QWS}\rangle_{i}$, where $\langle\cdots\rangle_{s}$ indicates integration only over the surface regions. Then, for even QWS's one has $\langle\cdots\rangle_{s}=-\langle\cdots\rangle_{i}$, while for odd QWS's both contributions add.

${ }^{32}$ Note that period doubling in the case of a contribution to SHG from both surface and interface results from the decomposition of the matrix elements in surface and interface contributions and not from the decomposition of $\chi$ into $\chi^{i}$ and $\chi^{s}$.

${ }^{33}$ QWS above and below $E_{F}$ might affect SHG differently due to matrix element and hybridization effects.

${ }^{34}$ N. E. Christensen and B. O. Seraphin, Phys. Rev. B 4, 3321 (1971). 
${ }^{35}$ Since the regions with large joint density of states are on the average at a $\mathbf{k}_{\perp}$ vector of $\frac{7}{10}$ of the Brillouin-zone length, this fixes the MOKE period at approximately $7 \mathrm{ML}$.

${ }^{36} \mathrm{~A}$ lower limit for the MOKE oscillation period is determined by the QWS's appearing at $E_{F}$. Thus the oscillation period is rather independent of $\hbar \omega$, although not as strict as in photoemission, since QWS's above $E_{F}$ can also be used as final states. 